\title{
Synthesis and Structural Study of Thiacyclophanes Utilizing Dibromides and Methane Dithiolate
}

\section{Perumal Rajakumar, ${ }^{* \dagger}$ Manickam Dhanasekaran, ${ }^{\dagger}$ Subramaniyan Selvam, ${ }^{\dagger}$ Paduthapillai Gopal Aravindan and Devadasan Velmurugan \\ Department of Organic Chemistry, Department of Crystallography and Biophysics, University of Madras, Guindy Campus, Chennai, India 600 025,}

perumalrajakumar@hotmail.com

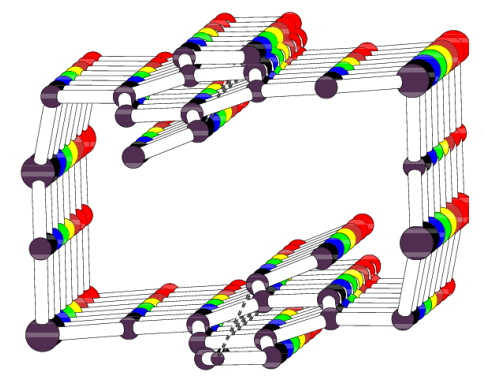

ABSTRACT: The synthesis of a series of thiacyclophanes and optically active binaphthol based chiral thiacyclophanes is reported with XRD structure. Two diastereomeric tetrathiacyclophanes are designed and synthesized. The two diastereomers are evidenced by crystal structure; the single-crystal X-ray studies revealed that one of the isomers to possesses an inherent property of self-assembling into vertical stack of tunnel-like structures.

\section{CONTENTS}

S2-S4 : $\quad$ General experimental procedure; spectroscopic and analytical data for reported cyclophanes.

S5 : : $\quad{ }^{1} \mathrm{H}$ NMR spectrum of Cyclophane 13.

S6 : $\quad$ Mass spectrum of Cyclophane 13.

S7-S9 : $\quad$ ORTEP diagram of Cyclophanes 6, 12, 13, 15 and 16 at $50 \%$ probability level.

S10 : $\quad$ Self-assembly views of cyclophane 15. 


\section{Supporting Information}

General Experimental Procedure: A mixture of dibromide (5 mmol) and $\mathrm{CS}_{2}(10$ mmol $)$ in dry THF $(75 \mathrm{~mL})$ was added to a slurry of $\mathrm{NaBH}_{4}(20 \mathrm{mmol})$ in THF $(25 \mathrm{~mL})$ at room temperature, and the resulting solution was refluxed for $6 \mathrm{~h}$. The reaction mixture was then quenched with aqueous ammonium chloride, solvent was evaporated under reduced pressure and crude product was extracted with $\mathrm{CHCl}_{3}(2 \times 100 \mathrm{~mL})$ and dried over sodium sulfate. The product was purified by column chromatography ( $\mathrm{SiO} 2)$ using ethyl acetate/hexane solvent mixture as eluent.

Cyclophanes 5: (27\%) as a colorless solid; mp 194-196 ${ }^{\circ} \mathrm{C}{ }^{1} \mathrm{H}$ NMR $\left(\mathrm{CDCl}_{3}\right) \delta 2.95(\mathrm{~s}, 2 \mathrm{H}), 3.63(\mathrm{~s}, 4 \mathrm{H}), 5.09(\mathrm{~s}, 4 \mathrm{H}), 6.48(\mathrm{~d}, 4 \mathrm{H}, J=8.6 \mathrm{~Hz})$, $6.89(\mathrm{~d}, 4 \mathrm{H}, J=8.6 \mathrm{~Hz}), 7.38-7.43(\mathrm{~m}, 4 \mathrm{H}) ;{ }^{13} \mathrm{C} \mathrm{NMR}\left(\mathrm{CDCl}_{3}\right) \delta 158.8$, 131.1, 130.9, 130.2, 129.0, 115.0, 114.1, 69.8, 37.9, 35.3; MS (EI) $(\mathrm{m} / z) 46$, 78, $104(100 \%), 138,195,208,309,347,394\left(\mathrm{M}^{+}\right)$; Anal. Calcd for $\mathrm{C}_{23} \mathrm{H}_{22} \mathrm{O}_{2} \mathrm{~S}_{2}$ : C, 70.02; H, 5.62. Found: C, 70.21; H, 5.76.

Cyclophanes 6: $(48 \%)$ as a colorless solid; $\mathrm{mp} 148-150{ }^{\circ} \mathrm{C}{ }^{1} \mathrm{H}$ NMR $\left(\mathrm{CDCl}_{3}\right) \delta 3.02(\mathrm{~s}, 2 \mathrm{H}), 3.58(\mathrm{~s}, 4 \mathrm{H}), 5.15(\mathrm{~s}, 4 \mathrm{H}), 6.64(\mathrm{~d}, 4 \mathrm{H}, J=8.3 \mathrm{~Hz})$, $6.89(\mathrm{~d}, 4 \mathrm{H}, J=8.3 \mathrm{~Hz}), 7.25(\mathrm{~d}, 2 \mathrm{H}, J=8.2 \mathrm{~Hz}), 7.35(\mathrm{t}, 1 \mathrm{H}, J=8.2 \mathrm{~Hz})$, 7.57 (s, $1 \mathrm{H}) ;{ }^{13} \mathrm{C} \mathrm{NMR}\left(\mathrm{CDCl}_{3}\right) \delta 156.7,137.9,129.6,129.0,126.5,126.3$, 115.2, 69.1, 35.0, 29.4; MS (EI) (m/z) 57, 78, 104 (100\%), 136, 209, 315, 348, $394\left(\mathrm{M}^{+}\right)$; Anal. Calcd for $\mathrm{C}_{23} \mathrm{H}_{22} \mathrm{O}_{2} \mathrm{~S}_{2}: \mathrm{C}, 70.02 ; \mathrm{H}, 5.62$. Found: $\mathrm{C}$, 70.18; H, 5.75 . 
Cyclophanes 7: (32\%) as a colorless solid; mp 196-198 ${ }^{\circ} \mathrm{C}{ }^{1} \mathrm{H}$ NMR $\left(\mathrm{CDCl}_{3}\right) \delta 3.03(\mathrm{~s}, 2 \mathrm{H}), 3.53(\mathrm{~s}, 4 \mathrm{H}), 5.19(\mathrm{~s}, 4 \mathrm{H}), 6.73(\mathrm{~d}, 4 \mathrm{H}, J=8.8 \mathrm{~Hz})$, $6.83(\mathrm{~d}, 4 \mathrm{H}, J=8.8 \mathrm{~Hz}), 7.30(\mathrm{~d}, 2 \mathrm{H}, J=7.8 \mathrm{~Hz}), 7.62(\mathrm{t}, 1 \mathrm{H}, J=7.8 \mathrm{~Hz})$; ${ }^{13} \mathrm{C}$ NMR $\left(\mathrm{CDCl}_{3}\right) \delta 156.8,156.7,135.7,129.8,129.6,122.3,114.9,70.2$, 34.9, 29.3; MS (EI) $(\mathrm{m} / z)$ 78, 105 (100\%), 213, 216, 316, 349, $395\left(\mathrm{M}^{+}\right)$; Anal. Calcd for $\mathrm{C}_{22} \mathrm{H}_{21} \mathrm{NO}_{2} \mathrm{~S}_{2}$ : C, 66.80; H, 5.35; N, 3.54. Found: C, 66.98; H, 5.47; N, 3.48 .

Cyclophanes 11: $(41 \%)$ as a colorless solid; mp $72-74{ }^{\circ} \mathrm{C}$; $[\alpha]_{D}^{30}=-$ 126.69, $\left(c 0.0175, \mathrm{CHCl}_{3}\right)^{1 \mathrm{H}} \mathrm{NMR}\left(\mathrm{CDCl}_{3}\right) \delta 3.04(\mathrm{~s}, 2 \mathrm{H}), 3.16(\mathrm{~d}, 2 \mathrm{H}, J=$ $13.5 \mathrm{~Hz}), 3.39$ (d, 2H, $J=13.5 \mathrm{~Hz}), 4.85(\mathrm{~d}, 2 \mathrm{H}, J=12.4 \mathrm{~Hz}), 4.91(\mathrm{~d}, 2 \mathrm{H}$, $J=12.4 \mathrm{~Hz}), 6.59(\mathrm{~s}, 2 \mathrm{H}), 6.84-7.31(\mathrm{~m}, 14 \mathrm{H}), 7.78(\mathrm{~d}, 2 \mathrm{H}, J=8.0 \mathrm{~Hz})$, $7.84(\mathrm{~d}, 2 \mathrm{H}, J=9.0 \mathrm{~Hz}) ;{ }^{13} \mathrm{C} \mathrm{NMR}\left(\mathrm{CDCl}_{3}\right) \delta 154.3,138.2,138.1,137.9$, $134.5,129.9,129.7,128.8,128.4,128.3,128.2,128.1,128.1,126.8,125.8$, 121.4, 71.6, 36.4, 33.4; MS (EI) (m/z) 58, 67, 105(100\%), 134, 164, 190, 205, 236, 268, 284, $570 \mathrm{M}^{+}$); Anal. Calcd for $\mathrm{C}_{37} \mathrm{H}_{30} \mathrm{O}_{2} \mathrm{~S}_{2}: \mathrm{C}, 77.86 ; \mathrm{H}, 5.30$. Found: C, 77.68; H, 5.44.

Cyclophanes 12: $(55 \%)$ as a colorless solid; mp 114-116 ${ }^{\circ} \mathrm{C} ;[\alpha]_{\mathrm{D}}^{30}=$ 146.30, $\left(c\right.$ 0.0175, $\left.\mathrm{CHCl}_{3}\right){ }^{1} \mathrm{H}$ NMR $\left(\mathrm{CDCl}_{3}\right) \delta 3.30(\mathrm{~s}, 2 \mathrm{H}), 3.53(\mathrm{~s}, 4 \mathrm{H})$, $5.04(\mathrm{~d}, 2 \mathrm{H}, J=13.2 \mathrm{~Hz}), 5.22(\mathrm{~d}, 2 \mathrm{H}, J=13.2 \mathrm{~Hz}), 6.67(\mathrm{~d}, 4 \mathrm{H}, J=8.0$ $\mathrm{Hz}), 6.91(\mathrm{~d}, 4 \mathrm{H}, J=8.0 \mathrm{~Hz}), 7.26-7.40(\mathrm{~m}, 8 \mathrm{H}),, 7.77(\mathrm{~d}, 2 \mathrm{H}, J=8.0 \mathrm{~Hz})$, $7.84(\mathrm{~d}, 2 \mathrm{H}, J=9.0 \mathrm{~Hz}) ;{ }^{13} \mathrm{C} \mathrm{NMR}\left(\mathrm{CDCl}_{3}\right) \delta 151.5,135.1,133.9,132.1$, 127.0, 127.0, 126.6, 125.8, 124.6, 124.3, 123.1, 121.5, 117.9, 113.0, 67.6, 33.1, 27.2; MS (EI) (m/z) 52, 77, 89 (100\%), 133, 185, 207, 218, 237, 286, $570 \mathrm{M}^{+}$); Anal. Calcd for $\mathrm{C}_{37} \mathrm{H}_{30} \mathrm{O}_{2} \mathrm{~S}_{2}$ : C, 77.86; H, 5.30. Found: $\mathrm{C}, 78.05$; $\mathrm{H}, 5.50$. 
Cyclophanes 13: (28\%) as a colorless solid; mp 194-196 ${ }^{\circ} \mathrm{C}{ }^{1} \mathrm{H}$ NMR $\left(\mathrm{CDCl}_{3}\right) \delta 3.36(\mathrm{~s}, 4 \mathrm{H}), 3.72(\mathrm{~s}, 8 \mathrm{H}), 6.95(\mathrm{~s}, 2 \mathrm{H}), 7.18-7.27(\mathrm{~m}, 6 \mathrm{H}),{ }^{13} \mathrm{C}$ NMR $\left(\mathrm{CDCl}_{3}\right) \delta 36.1,38.1,127.6,128.4,129.5,138.3$; MS (EI) $(\mathrm{m} / z) 105$ (100\%), 135, 166, 198, 227, $364\left(\mathrm{M}^{+}\right)$; Anal. Calcd for $\mathrm{C}_{18} \mathrm{H}_{20} \mathrm{~S}_{4}$ : C, 59.29; H, 5.53. Found: C, 59.57; H, 5.71.

Cyclophanes 15: (27\%) as a colorless solid; mp 222-225 ${ }^{\circ} \mathrm{C}{ }^{1} \mathrm{H}$ NMR $\left(\mathrm{CDCl}_{3}\right) \delta 2.98(\mathrm{~d}, 2 \mathrm{H}, J=11.2 \mathrm{~Hz}), 3.27(\mathrm{~d}, 2 \mathrm{H}, J=11.2 \mathrm{~Hz}), 3.35(\mathrm{~d}, 4 \mathrm{H}$, $J=13.6 \mathrm{~Hz}), 3.71(\mathrm{~s}, 12 \mathrm{H}), 4.15(\mathrm{~d}, 4 \mathrm{H}, J=13.6 \mathrm{~Hz}), 6.67(\mathrm{~s}, 4 \mathrm{H}) ;{ }^{13} \mathrm{C}$ $\operatorname{NMR}\left(\mathrm{CDCl}_{3}\right) \delta 151.2,125.1,113.6,56.3,30.4,29.6 ; \mathrm{MS}(\mathrm{EI})(\mathrm{m} / \mathrm{z}) 46,91$, 135, 164 (100\%), 209, 242, 274, 319, 392, 438, $484\left(\mathrm{M}^{+}\right)$; Anal. Calcd for $\mathrm{C}_{22} \mathrm{H}_{28} \mathrm{O}_{4} \mathrm{~S}_{4}$ : C, 54.51; H, 5.82. Found: C, 54.40; H, 5.76.

Cyclophanes 16: (18\%) as a colorless solid; mp 206-208 ${ }^{\circ} \mathrm{C}{ }^{1} \mathrm{H}$ NMR $\left(\mathrm{CDCl}_{3}\right) \delta 3.15(\mathrm{~s}, 4 \mathrm{H}), 3.31(\mathrm{~d}, 4 \mathrm{H}, J=14 \mathrm{~Hz}), 3.72(\mathrm{~s}, 12 \mathrm{H}), 4.13(\mathrm{~d}, 4 \mathrm{H}$, $J=14 \mathrm{~Hz}), 6.75(\mathrm{~s}, 4 \mathrm{H}) ;{ }^{13} \mathrm{C} \mathrm{NMR}\left(\mathrm{CDCl}_{3}\right) \delta 151.4,124.9,113.0,56.3$, 30.2, 29.7; MS (EI) (m/z) 49, 91, 134, 165 (100\%), 195, 242, 287, 328, 360, $484\left(\mathrm{M}^{+}\right)$; Anal. Calcd for $\mathrm{C}_{22} \mathrm{H}_{28} \mathrm{O}_{4} \mathrm{~S}_{4}$ : C, 54.51; H, 5.82. Found: C, 54.75; H, 5.97. 


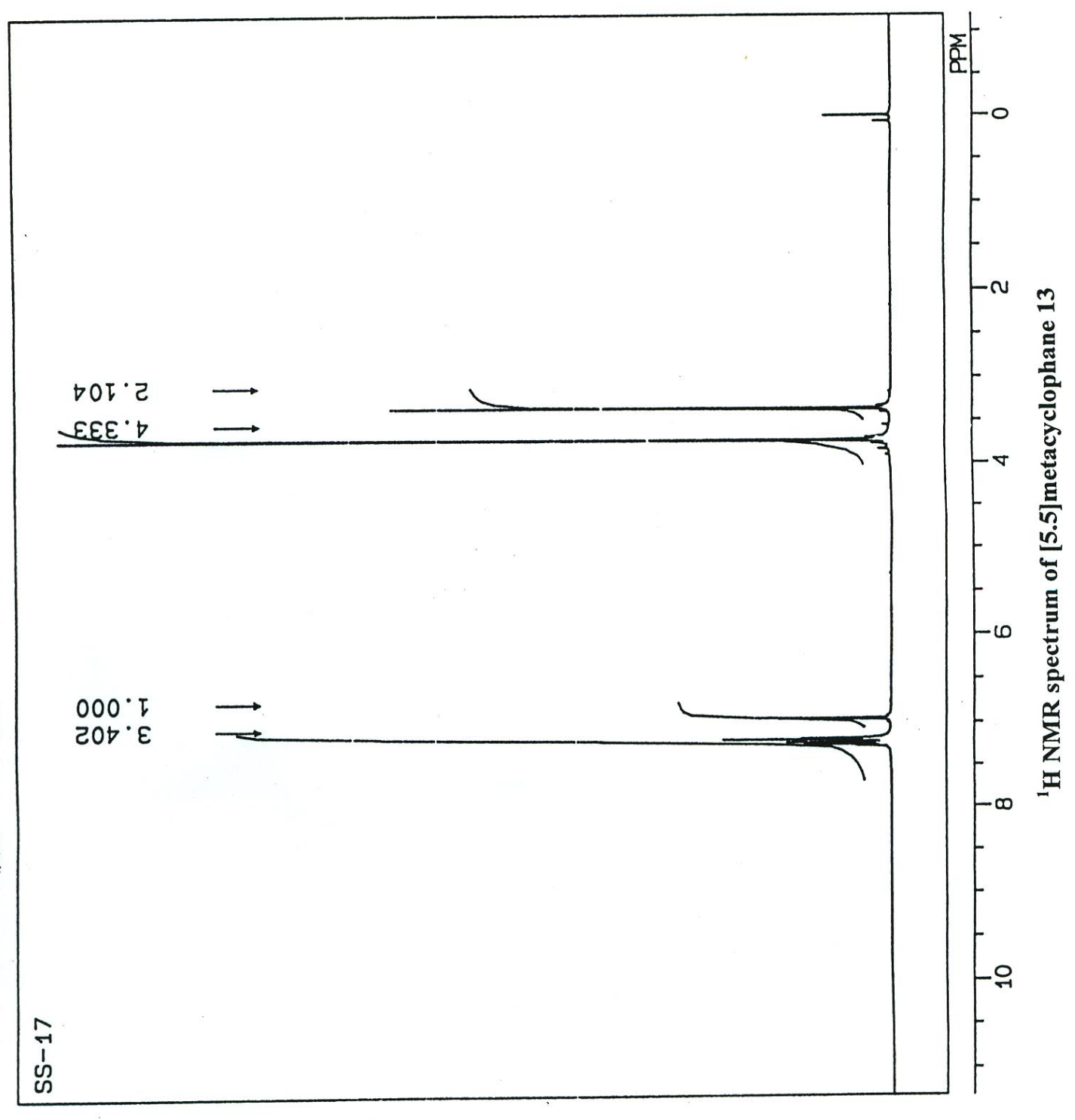


$\stackrel{\text { OC }}{12 / 1 / 04 \text { 1:11:05 PM }}$

Date Run: 12-01-2004

File: MDSS17A

Sample:

Instrument: JEOL DX-303

Inlet: My Inlet
Ionization mode: EI+

Page 1

Time Run: 13:06:59

Run By: Mu

Printed by: Mu

Scan: 71

Base: $\mathrm{m} / \mathrm{z}$ 105; $28.3 \% F S$ TIC: 94759

R.T.: 2:41

\#Ions: 14

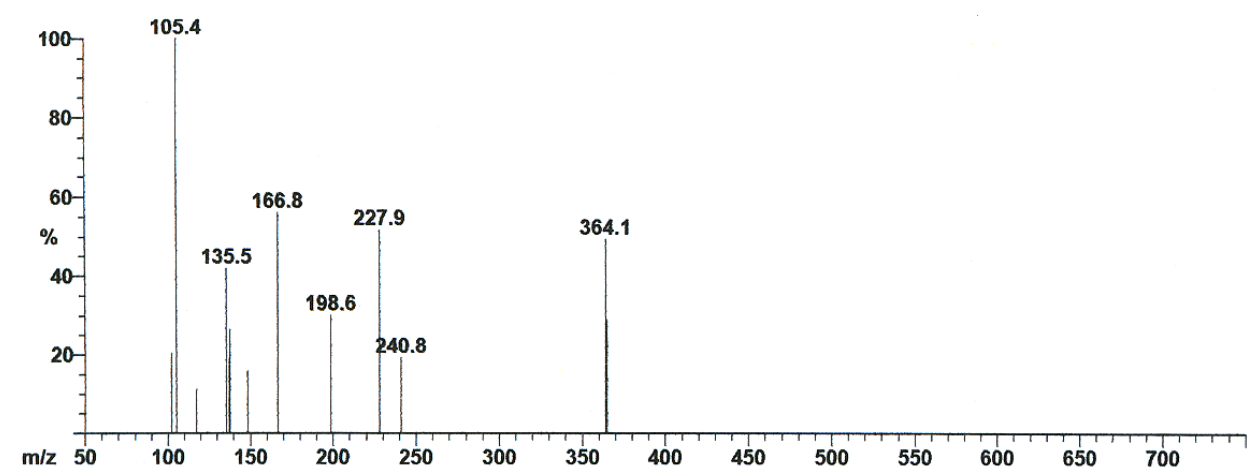

Threshold: 3\% of Base

Displayed TIC: 94759

\begin{tabular}{|c|c|c|c|c|c|c|c|}
\hline Mass & \%Base & Mass & $\%$ Base & Mass & $\%$ Base & Mass & $\%$ Base \\
\hline 102.4655 & 20.4 & 137.3787 & 19.1 & 198.5780 & 30.1 & 364.8701 & 28.7 \\
\hline 105.3843 & 100.0 & 137.5130 & 26.5 & 227.8803 & 51.7 & & \\
\hline 117.4187 & 11.1 & 148.5138 & 15.9 & 240.8390 & 19.3 & & \\
\hline 135.4816 & 42.0 & 166.7606 & 56.0 & 364.0508 & 49.4 & & \\
\hline
\end{tabular}

Mass spectrum of [5.5]metacyclophane 13 


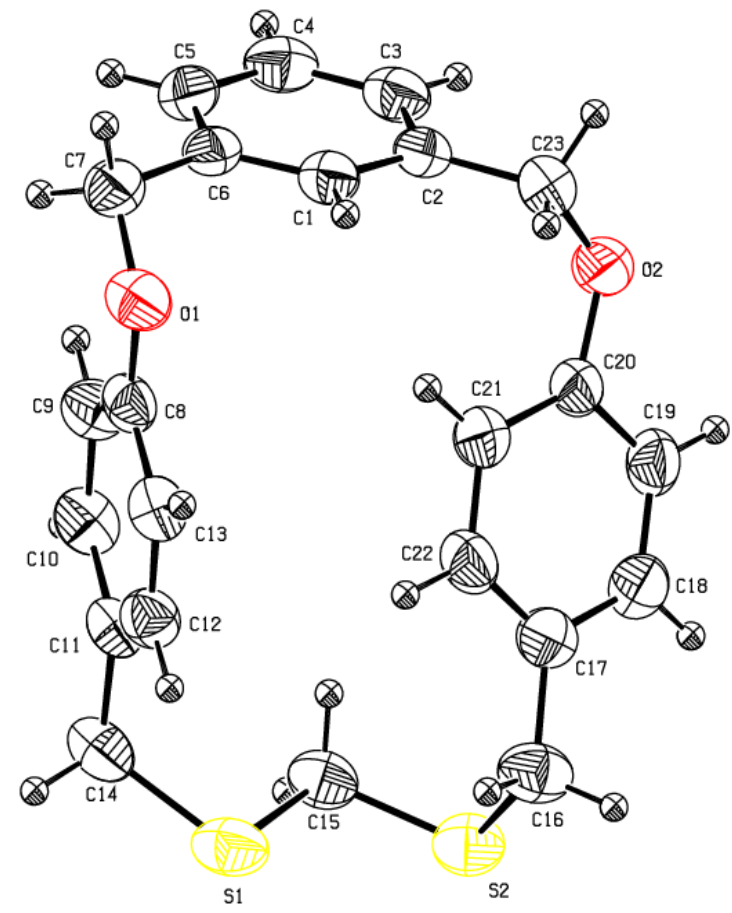

ORTEP drawing of cyclophane 6 showing $50 \%$ probability level. 


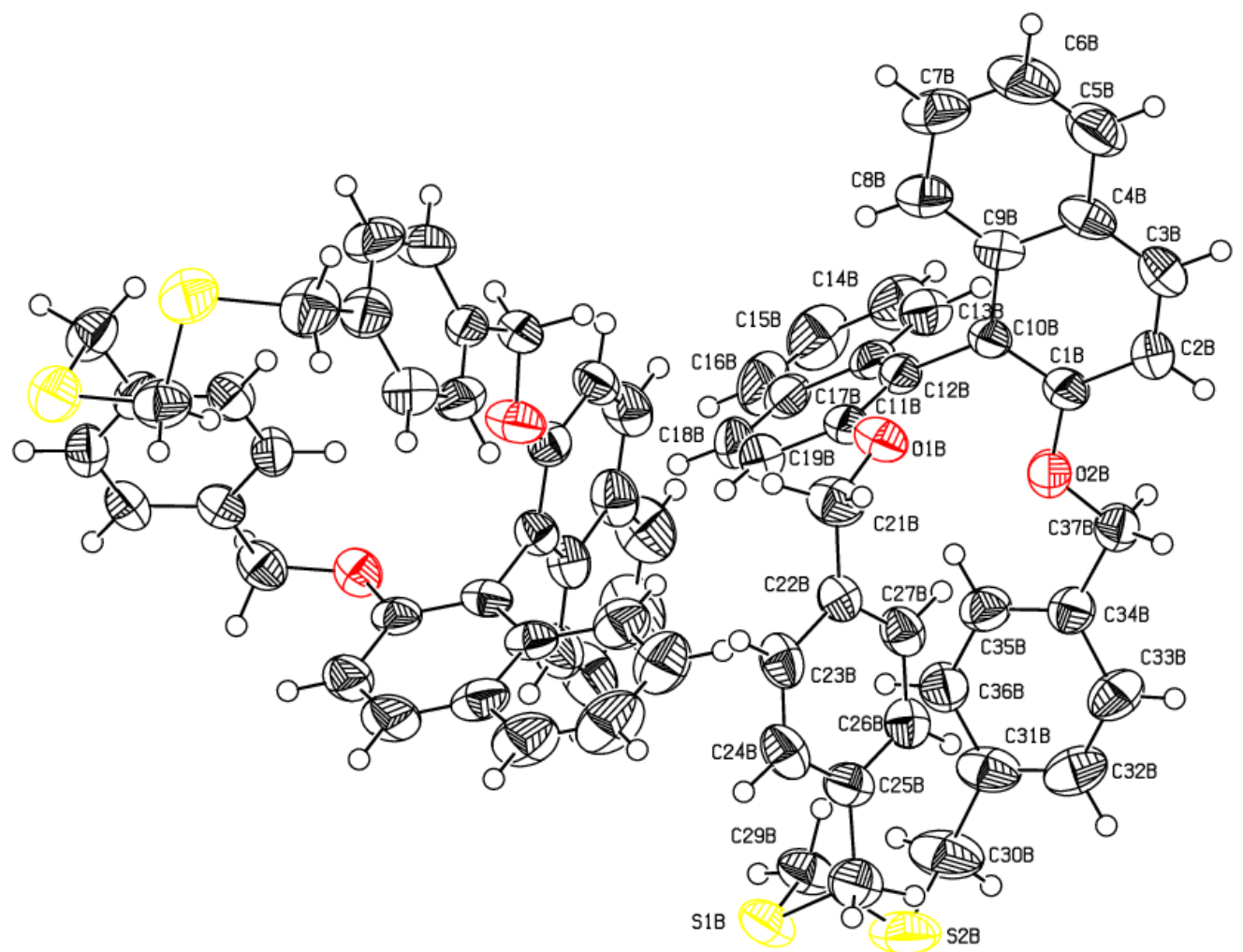

ORTEP diagram of cyclophane 12 showing $50 \%$ probability level.

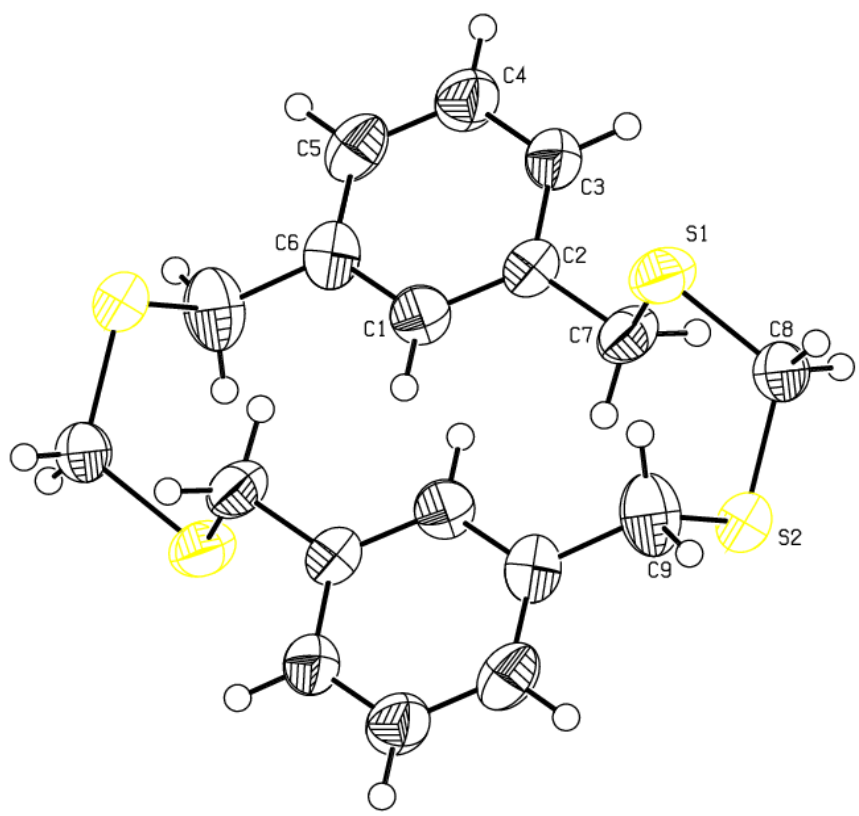

ORTEP drawing of cyclophane 13 showing $50 \%$ probability level. 


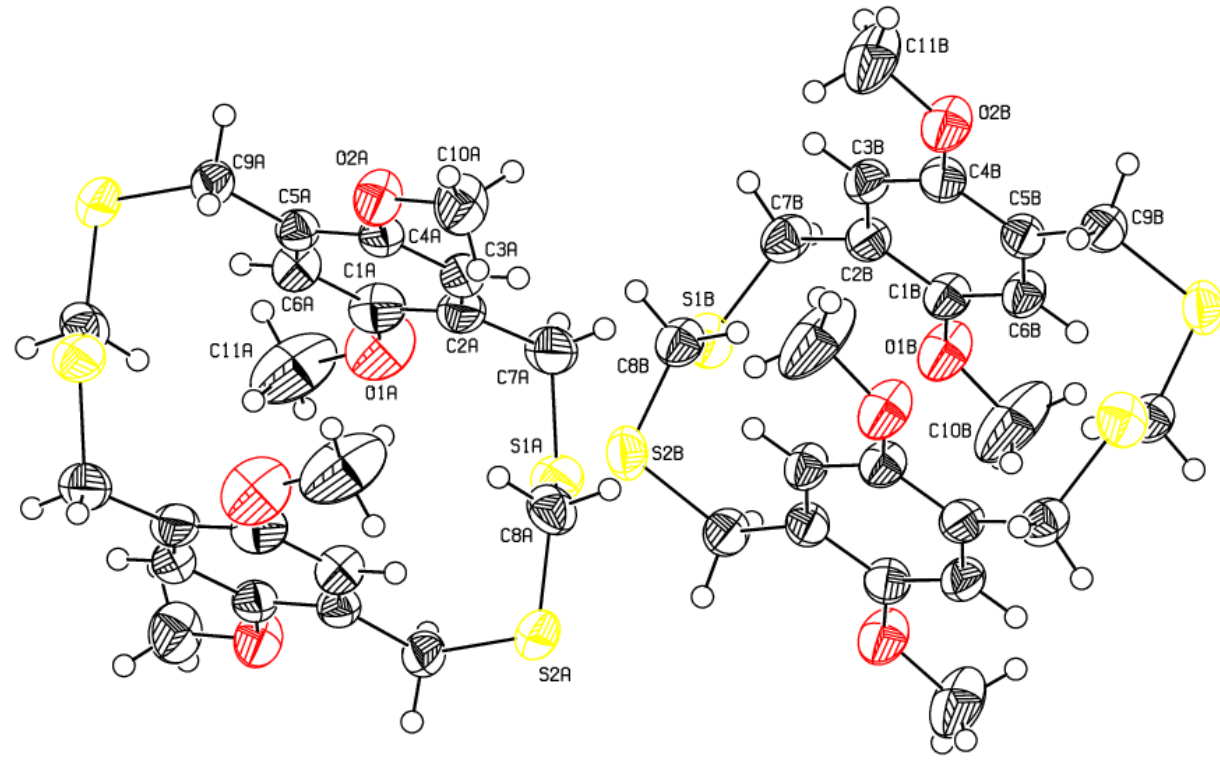

ORTEP diagram of cyclophane 15 showing $50 \%$ probability level.

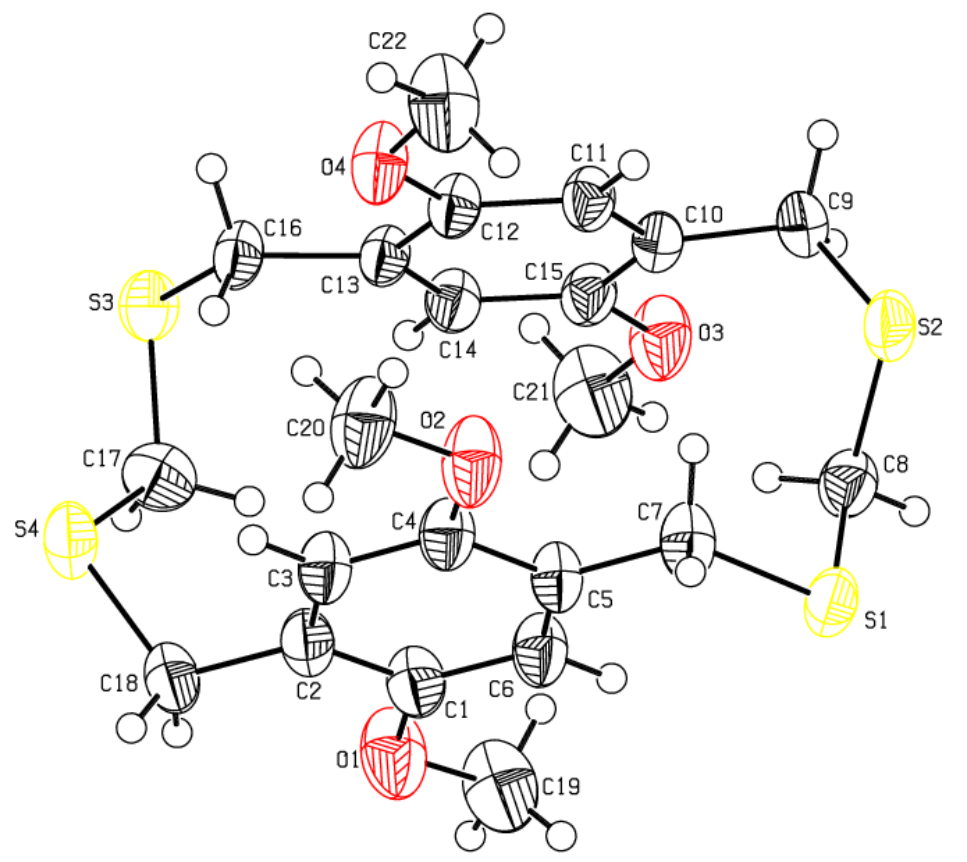

ORTEP drawing of cyclophane 16 showing $50 \%$ probability 
Self assembly views of cyclophane 15

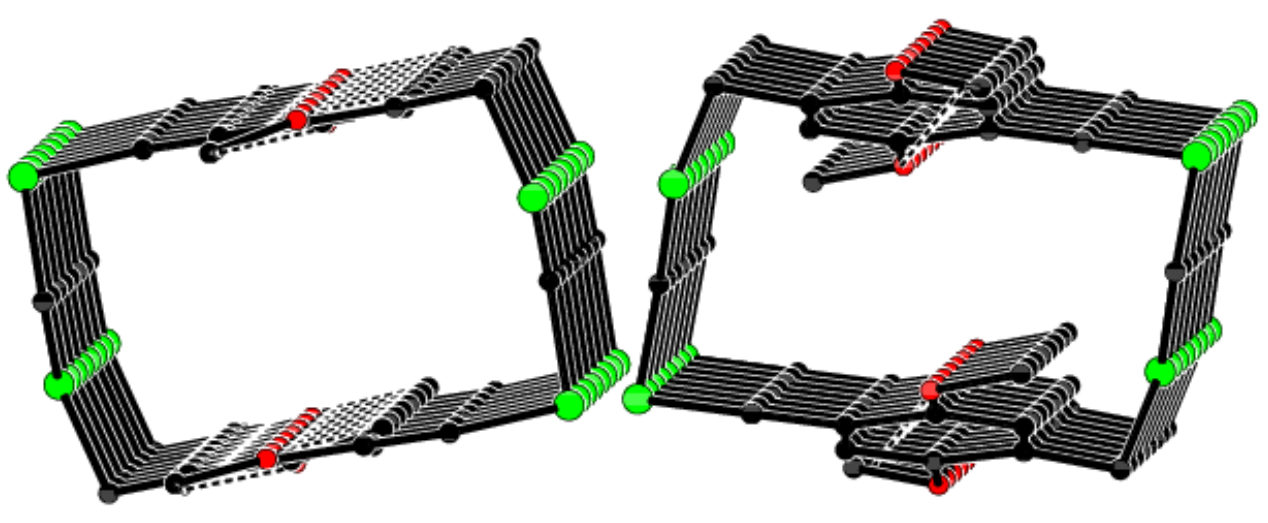

Front view of cyclophane 15

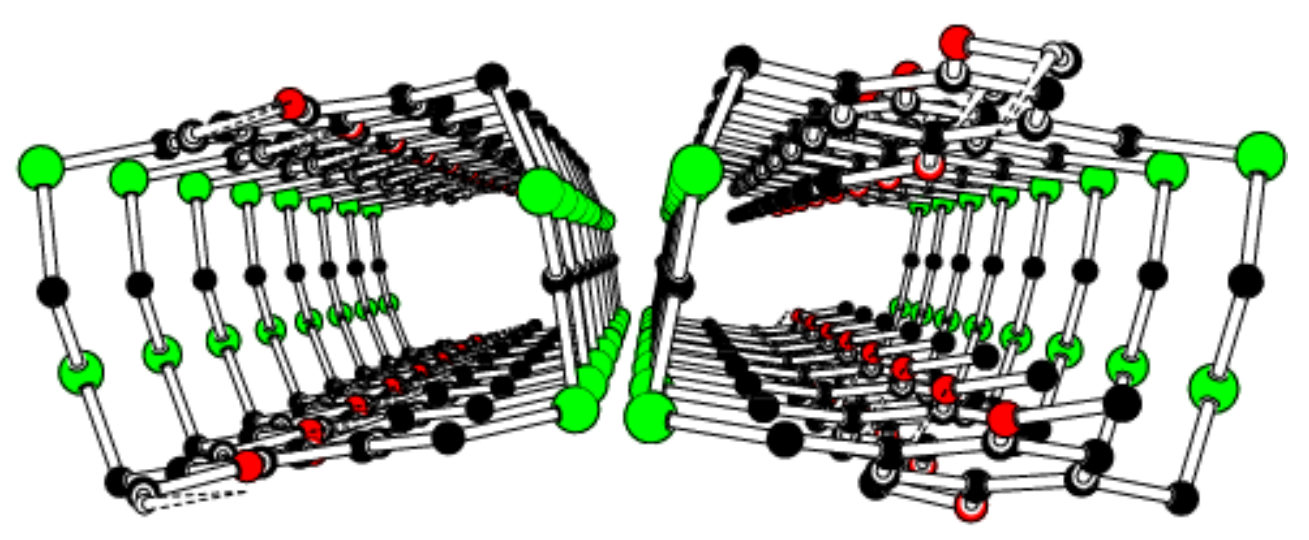

Perspective view of cyclophane 15

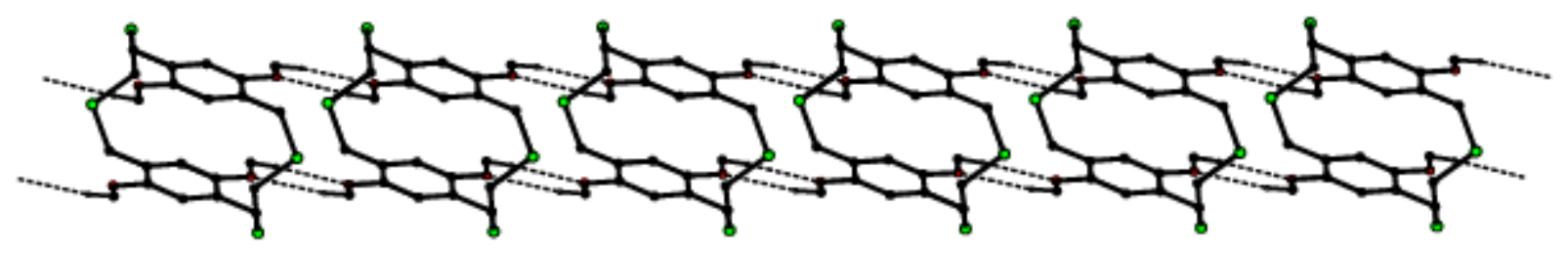

Linear view (chain) of cyclophane 15 